International Journal of Pure and Applied Mathematics

Volume 103 No. 3 2015, 477-483

ISSN: 1311-8080 (printed version); ISSN: 1314-3395 (on-line version)

url: http://www.ijpam.eu

doi: http://dx.doi.org/10.12732/ijpam.v103i3.9

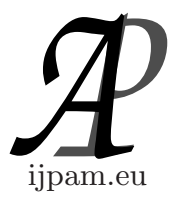

\title{
INVERSE DOMINATION NUMBER OF JUMP GRAPH
}

\author{
M. Karthikeyan ${ }^{1 \S}$, A. Elumalai ${ }^{2}$ \\ ${ }^{1}$ Bharathiar University \\ Coimbatore, 641046, INDIA \\ ${ }^{1}$ Department of Mathematics \\ Agni College of Technology \\ Chennai, 600130, INDIA \\ ${ }^{2}$ Department of Mathematics \\ Valliammai Engineering College \\ Chennai, 603203, INDIA
}

\begin{abstract}
Let $J(G)=(V, E)$ be a jump graph. Let $D$ be a minimum dominating set in a jump Graph $J(G)$. If $V-D$ contains a dominating set $D^{\prime}$ of $J(G)$, then $D^{\prime}$ is called an inverse dominating set with respect to $D$. The minimum cardinality of an inverse dominating set of a Jump graph $J(G)$ is called the inverse domination number of $J(G)$. In this paper We study the graph theoretic properties of inverse domination of Jump graph and its exact values for some standard graphs. The relation between inverse domination of Jump graph with other parameters is also investigated.
\end{abstract}

AMS Subject Classification: $05 \mathrm{C} 78$

Key Words: graph, circumference, diameter, domination, inverse domination number, jump graph

\section{Introduction}

Let $G(p, q)$ be a graph with $p=|V|$ and $q=|E|$ denote the number of vertices and edges of a graph $G$ respectively. All the graphs considered here are finite,

Received: April 30, 2015

(c) 2015 Academic Publications, Ltd.

$\S$ Correspondence author url: www.acadpubl.eu 
non-trivial, undirected and connected without loops or multiple edges. For basic terminology, we refer to Chartrand and Lesniak [7]. A set $D$ of vertices in a graph $G=(V, E)$ is a dominating set if every vertex in $V-D$ is adjacent to some vertex in $D$. The domination number $\gamma(G)$ of $G$ is the minimum cardinality of a dominating set of $G$. Let $D$ be a minimum dominating set of $G$. If $V-D$ contains a dominating set $D^{\prime}$ of $G$, then $D^{\prime}$ is called an inverse dominating set with respect to $D$. The inverse domination number $\gamma^{-1}(G)$ of $G$ is the minimum cardinality of an inverse dominating set of $G$. This concept was first introduced by Kulli and Sigarkanthi [3] and it was studied by several graph theorists in $[8,9]$.

In general the degree of vertex $v$ in a graph $G$ is the number of edges of $G$ incident with $v$ and it is denoted by deg $v$. The maximum (minimum) degree among the vertices of $G$ is denoted by $\Delta(G)(\delta(G))$. We denote the minimum number of edges in edge cover of $G$ (i.e., edge cover number) by $\alpha_{1}(G)$ and the minimum number of edges in independent set of edges of $G$ (i.e., edge independent set) by $\beta_{1}(G)$. The subgraph induced by $X \subseteq V$ is denoted by $\langle X\rangle$. A vertex of degree one is called an pendent vertex. A vertex adjacent to pendent vertex is called the support vertex. The maximum $d(u, v)$ for all $u$ in $G$ is eccentricity of $v$ and the maximum eccentricity is the diameter $\operatorname{diam}(G)$. The circumference of a graph $G$ with atleast one cycle is the length of a longest cycle in $G$ and it is denoted by $\operatorname{circum}(G)$.). As usual $P_{n}, C_{n}$ and $K_{n}$ are respectively, the path, cycle and complete graph of order $n, K_{r, s}$ is the complete bipartite graph with two partite sets containing $r$ and $s$ vertices. Any undefined term or notation in this paper can be found in [2].

Definition 1. The line graph $L(G)$ of $G$ has the edges of $G$ as its vertices which are adjacent in $L(G)$ if and only if the corresponding edges are adjacent in $G$. We call the complement of line graph $L(G)$ as the jump graph $J(G)$ of $G$, found in [4]. The jump graph $J(G)$ of a graph $G$ is the graph defined on $E(G)$ and in which two vertices are adjacent if and only if they are not adjacent in $G$. Since both $L(G)$ and $J(G)$ are defined on the edge set of a graph $G$.

Remark 2. The isolated vertices of $G$ (if $G$ has) play no role in line graph and jump graph transformation. Here we assume that the graph $G$ under consideration is non-empty and has no isolated vertices found in [4].

Definition 3. Let $G=(V, E)$ be a graph. Let $D$ be a minimum dominating set in a Graph $G$. If $V-D$ Contains a dominating set $D^{\prime}$ of $G$, then $D^{\prime}$ is called an inverse dominating set with respect to $D$ the minimum cardinality of an inverse dominating set of a graph $G$ is called the inverse domination number of $G$ and it is denoted by $\gamma^{-1}(G)$ studied in $[3,6]$. 


\section{Example 4.}

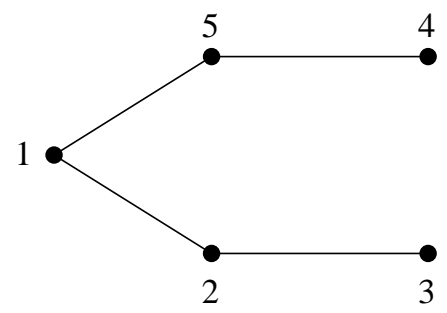

Figure 1

$D_{1}=\{2,5\}, D_{2}=\{2,4\}, D_{3}=\{3,5\}$ are the minimum dominating sets. Their corresponding inverse dominating sets are $D_{1}^{\prime}=\{1,3,4\}, D_{2}^{\prime}=\{3,5\}$, $D_{3}^{\prime}=\{2,4\}$ respectively. Thus $\gamma(G)=2$ is the domination number of $G$. $\gamma^{-1}(G)=2$ is the inverse domination number of $G$.

Remark 5. Every graph without isolated vertices contains an inverse dominating set, since the complement of any minimal dominating set is also a dominating set. Thus we consider a graph without isolated vertices.

Definition 6. Let $J(G)=(V, E)$ be a jump graph. Let $D$ be a minimum dominating set in a jump Graph $J(G)$. If $V-D$ Contains a dominating set $D^{\prime}$ of $J(G)$, then $D^{\prime}$ is called an inverse dominating set with respect to $D$. The minimum cardinality of an inverse dominating set of a jump graph $J(G)$ is called the inverse domination number of $J(G)$ and it is denoted by $\gamma^{-1}[J(G)]$.

For any graph $G$, with $p \leq 4$, the jump graph $J(G)$ of $G$, is disconnected. Since we study only the connected jump graph, we choose $p>4$ [5].

We recall following classical theorems to prove our results.

Theorem 7. [1] Let $G$ be a graph without isolated vertices. If $D$ is a minimal dominating set, then $V-D$ is a dominating set.

Theorem 8. [3] If a graph $G$ has no isolated vertices, then $\gamma(G) \leq \gamma^{-1}(G)$.

Theorem 9. [3] If a graph $G$ has no isolated vertices, then $\gamma(G)+$ $\gamma^{-1}(G) \leq p$

\section{Main Results}

\section{Theorem 10.}

1. For any path $P_{p}$ with $p \geq 5 \gamma^{-1}\left[J\left(P_{p}\right)\right]=2$ 
2. For any Cycle $C_{p}$, with $p \geq 5 \gamma^{-1}\left[J\left(C_{p}\right)\right]=2$

3. For any complete graph $K_{p}$, with $p \geq 5 \gamma^{-1}\left[J\left(K_{p}\right)\right] \geq 2$

4. For any complete bipartite graph $K_{m n}$

$$
\gamma^{-1}\left[J\left(K_{m n}\right)\right]= \begin{cases}2 & \text { for } k_{2, n} \text { where } n \geq 2 \\ 3 & \text { for } k_{m, n} \text { where } m, n \geq 3\end{cases}
$$

5. For any wheel $W_{p}, \gamma^{-1}\left[J\left(W_{p}\right)\right]= \begin{cases}3 & \text { if } p=5,6 \\ 3 & \text { if } p \geq 7\end{cases}$

Theorem 11. For any connected graph $G \gamma^{-1}[J(G)] \geq 2$.

Proof of the theorem is obvious

Theorem 12. For any connected $(p, q)$ graph $\gamma^{-1}[J(G)] \leq q-\beta_{1}(G)+1$

Proof. Let $V-D=\left\{v_{1}, v_{2}, v_{3}, \ldots, v_{n}\right\}$ be the set of vertices in $J(G)$ corresponding to the set of independent edges $\left\{e_{1}, e_{2}, e_{3}, \ldots, e_{n}\right\}$ of $G$. By the definition of $J(G)$, the elements of $V-D$ form an induced subgraph $\left\langle k_{n}\right\rangle$ in $J(G)$. Further, let $S \cup\left\{v_{1}\right\}$, where $S \subset[V[J(G)]-(V-D)]$ be an inverse dominating set in $J(G)$. It follows that $\left|S \cup\left\{v_{1}\right\}\right| \leq|V[J(G)]-(V-D)|+\left|v_{1}\right|$ therefore $\gamma^{-1}[J(G)] \leq q-\beta_{1}(G)+1$

Theorem 13. For any connected graph $G$ with diameter, $\operatorname{diam}(G) \geq 2$, $\gamma^{-1}[J(G)] \geq 2$

Proof. Let $u v$ be a path of maximum distance in $G$. Then $d(u, v)=$ $\operatorname{diam}(G)$ We can prove the theorem with the following cases.

Case (i): For $\operatorname{diam}(G)=2$, choose a vertex $v_{1}$ of eccentricity 2 with maximum degree among others. Let $V-D=\left\{v_{1}, v_{2}, \ldots\right\}$ corresponding to the elements of $\left\{e_{1}, e_{2}, \ldots\right\}$ forming an inverse dominating set in jump graph $J(G)$. Every vertex $u \notin V-D$ is adjacent to a vertex in $V-D$. Hence $V-D$ is a minimum inverse dominating set. So the inverse domination number of the jump graph will be equal to the degree of $v_{1}$ also by Theorem 11 , we say $\gamma^{-1}[J(G)]>2$

Case (ii): For $\operatorname{diam}(G)>2$. Let $e_{1}$ be any edge adjacent to $u$ and $e_{2}$ be any edge adjacent to $v$. Let $\left\{e_{1}, e_{2}\right\} \subseteq E(G)$ form a corresponding vertex set $\left\{v_{1}, v_{2}\right\} \subseteq V(J(G))$. These two vertices form an inverse dominating set in jump graph. Since these vertices $\left\{v_{1}, v_{2}\right\}$ are adjacent to all other vertices of 
$V-D(J(G))$, it follows that $\left\{v_{1}, v_{2}\right\}$ becomes a minimum inverse dominating set. Hence $\gamma^{-1}[J(G)]=2$.

In view of above cases, we can conclude that for any connected graph $G$, $\gamma^{-1}[J(G)] \geq 2$

Theorem 14. For any connected graph $G$ with circumference, $\operatorname{circum}(G)$ $\geq 4, \gamma^{-1}[J(G)] \geq 2$

Proof. Let $u-v-u$ be a longest cycle in $G$. Then $C(G)=\operatorname{Circum}(G)$. We can prove the theorem with the following cases.

Case (i): For $\operatorname{Circum}(G)=4$, choose a vertex $v_{1}$ that have longest cycle among others. Let $V-D=\left\{v_{1}, v_{2}, \ldots\right\}$ corresponding to the elements of $\left\{e_{1}, e_{2}, \ldots\right\}$ forming an inverse dominating set in jump graph $J(G)$. Every vertex $u \notin V-D$ is adjacent to a vertex in $V-D$. Hence $V-D$ is a minimum inverse dominating set. So the inverse domination number of the jump graph will be equal to the longest cycle also by Theorem 11, we say $\gamma^{-1}[J(G)]>2$.

Case (ii): For $\operatorname{Cirum}(G)>4$. Let $e_{1}$ be any edge adjacent to $u$ and $e_{2}$ be any edge adjacent to $v$. Let $\left\{e_{1}, e_{2}\right\} \subseteq E(G)$ form a corresponding vertex set $\left\{v_{1}, v_{2}\right\} \subseteq V(J(G))$. These two vertices form an inverse dominating set in jump graph. Since these vertices $\left\{v_{1}, v_{2}\right\}$ are adjacent to all other vertices of $V-D(J(G))$, it follows that $\left\{v_{1}, v_{2}\right\}$ becomes a minimum inverse dominating set. Hence $\gamma^{-1}[J(G)]=2$.

In view of above cases, we can conclude that for any connected graph $G$, $\gamma^{-1}[J(G)] \geq 2$

Theorem 15. For any tree $T$ with diameter greater than $3, \gamma^{-1}[J(T)] \geq 2$

Proof. If the diameter is less than or equal to 3 , then the jump graph will be disconnected.

Let $u v$ be a path of maximum length in a tree $T$ where diameter is greater than 3. Let $e_{i}$ be the pendant edge adjacent to $u$ and $e_{k}$ be the pendant edge adjacent to $v$. The vertex set $\left\{v_{i}, v_{k}\right\}$ of $J(T)$ corresponding to the edges of $\left\{e_{i}, e_{k}\right\}$ in $T$ will form an inverse dominating set in $J(T)$. Since all the other vertices of $V[J(T)]$ are adjacent with $\left\{v_{i}, v_{k}\right\}$, it forms a minimum inverse dominating set. Hence $\gamma^{-1}[J(T)] \geq 2$.

Theorem 16. For any connected $(p, q)$ graph $G, \gamma^{-1}[J(G)]<q-\Delta(G)$ where $\Delta(G)$ is the maximum degree of $G$. 
Proof. Let $V-D=\left\{v_{1}, v_{2}, v_{3}, \ldots, v_{n}\right\}$ be the set of vertices in $G$ and $V_{1}=(V-D)-v_{1}$ where $v_{1}$ is one of the vertices with maximum degree. By definition of jump graph, $E(G)=V[J(G)]$. Consider $I=\left\{e_{1}, e_{2}, e_{3}, \ldots, e_{k}\right\}$ as the set of edges adjacent to $v_{1}$ in $G$. Let $H \subseteq V(J(G))$ be the set of vertices of $J(G)$ such that, $H \subseteq E-I$. Then $H$ itself forms a minimally inverse dominating set. Therefore $\gamma^{-1}[J(G)] \leq|E|-|I|$. Hence $\gamma^{-1}[J(G)]<q-\Delta(G)$.

Theorem 17. For any connected graph $G$ without pendant vertex

$$
\gamma^{-1}[J(G)] \leq \delta(G)
$$

Proof. Let $V-D=\left\{v_{1}, v_{2}, v_{3}, \ldots, v_{n}\right\}$ be the set of vertices in $G$ and $v_{1}$ be one among the vertices with minimum degree. Let $\left\{e_{1}, e_{2}, e_{3}, \ldots, e_{k}\right\}$ be the set of edges adjacent to $v_{1}$ in $G$. Then $E_{1} \subseteq V[J(G)]$ will form the inverse dominating set in $J(G)$. So $\left|E_{1}\right|=\delta(G)$ obviously it becomes the minimum inverse dominating set. Therefore $\gamma^{-1}[J(G)] \leq \delta(G)$.

\section{Conclusion}

Thus we conclude the inverse domination number and the parameters of inverse domination number of jump graphs like $k_{p}, C_{p}, P_{p}, W_{p}$ and $K_{m, n}$.

\section{References}

[1] O. Ore, Theory of Graphs, Amer. Math. Soc. Colloq. Public., 38, providence (1962).

[2] F. Harary, Graph Theory, Addision-Wesley, Reading Mass. (1969).

[3] V.R. Kulli and S.C. Sigarkanti, Inverse domination in Graphs, Nat. Acad. Sci. Lett., 14 (1991), 473-475.

[4] G. Chartrand, H. Hevia, E.B. Jarrett, M. Schultz, Subgraph distances in graphs defined by edge transfers, Discrete Math., 170 (1997), 63-79.

[5] T.H. Haynes, S.T. Hedetniemi and P.J. Slater, Fundamentals of Domination in graphs, Marcel Dekker Inc, New York (1998).

[6] G.S. Domke, J.E. Dunbar and L.R. Markus, The inverse domination number of a graph, Ars. Combin., 72 (2004) 149-160. 
[7] G. Chartrand and L. Lesniak, Graphs and Digraphs, CRC (2004).

[8] P.D Johnson Jr., D.R. Prier and M. Walsh, On a problem of Domke, Haynes, Hedetniemi and Markus, Concerning the inverse domination number, AKCEJ. Graphs Combin., 7(2) (2010), 217-222.

[9] A. Frendrup, M.A. Henning, B. Randerath and P.D Vestergoard, On Conjecture about inverse domination in Graphs, Ars Combinatoria, 95A (2010), 103-111. 
